\title{
Von Aktivismus, Geographien und dem Dazwischen - Überlegungen anhand der Praxis von Kollektiv Orangotango
}

\author{
Severin Halder ${ }^{1}$ (iD $\cdot$ Paul Schweizer ${ }^{2}$ (iD \\ Eingegangen: 17. Mai 2020 / Überarbeitet: 13. Oktober 2020 / Angenommen: 15. Oktober 2020 / Online publiziert: 9. November 2020 \\ (c) Deutscher Verband für Angewandte Geographie (DVAG) 2020
}

\section{Zusammenfassung}

Dieser Artikel befasst sich mit dem Verhältnis von politischem Aktivismus und kritischer Forschung. Dabei führen wir in methodologische Ansätze der Aktionsforschung und der militanten Untersuchung sowie Diskussionen um aktivistische oder aktionsorientierte Geographie ein, die in der deutschsprachigen Geographie bislang wenig Beachtung finden. Diese Debatten ergänzen wir um die in lateinamerikanischen sozialen Bewegungen praktizierte - von Paulo Freires ,educação popular“ geprägte - Pädagogisierung des Politischen. Wie Aktionsforschung und militante Untersuchung fordert Freires „Bildung von unten“ die „Auflösung der Trennung“ zwischen Bildung und sozialer Transformation sowie die Einbeziehung anderer Wissensformen in kollektive „dialogische“ Bildungsprozesse. Die Praxis von Kollektiv Orangotango entsteht im Überschneidungsfeld von Subkultur, Forschung und Aktivismus. Anhand dieser Erfahrungen zeigen wir, wie eine als „Bildung von unten“ verstandene angewandte kritische Geographie die Trennung zwischen Forschung, Lernen und Aktivismus sowie den jeweiligen Akteuren und Akteurinnen verschwimmen lässt.

Schlüsselwörter Aktionsforschung $\cdot$ Militante Untersuchung $\cdot$ Soziale Bewegungen $\cdot$ Subkultur $\cdot$ Bildung von unten

\begin{abstract}
This article deals with the relationship between political activism and critical research. We introduce the methodological approaches of action research and militant investigation as well as discussions on activist or action-oriented geography, which have received little attention in the field of German-language geography so far. We supplement these debates with the pedagogising of the political as practiced in Latin American social movements, which was influenced by Paulo Freire's educação popular. Like action research and militant investigation, Freire's popular education calls for the dissolution of the division between education and social transformation and the inclusion of other forms of knowledge in collective "dialogical" educational processes. The practice of Kollektiv Orangotango arises in the intersection of subculture, research, and activism. On the basis of these experiences, we show how an applied critical geography, understood as popular education, blurs the separation between research and activism as well as between researchers, learners, and activists.
\end{abstract}

Keywords Action research $\cdot$ Militant investigation $\cdot$ Social movements $\cdot$ Subculture $\cdot$ Popular education

\section{Geographie und Aktivismus}

Kollektiv Orangotango entsteht seit den 2000er-Jahren im freundschaftlichen Umfeld kritischer Geographen und Geo-

Severin Halder

severinhalder@gmail.com, info@ orangotango.info

Paul Schweizer

schweizer@autistici.org

1 kollektiv orangotango, Okerstraße 12, 12049 Berlin, Deutschland

2 Aischbachstraße 2, 72070 Tübingen, Deutschland graphinnen, aus der Überschneidung unserer subkulturellen Sozialisation - in Hip-Hop- bzw. Soundsystem-Kultur, autonomen Zentren, globalisierungskritischen Bewegungen einerseits und der Begegnung mit politischer Basisarbeit und aktivistischer Lehr- und Forschungspraxis in lateinamerikanischen Kontexten andererseits.

Dieser Artikel befasst sich mit dem Verhältnis von politischem Aktivismus und kritischer Forschung. Dabei führen 
wir in methodologische Ansätze der Aktionsforschung und der militanten Untersuchung ein, die in der deutschsprachigen Geographie bislang wenig Beachtung finden. Anschließend zeigen wir anhand unserer Erfahrung mit Kollektiv Orangotango, wie eine, als „Bildung von unten“ verstandene, angewandte kritische Geographie die Trennung zwischen Forschung, Lernen und Aktivismus sowie den jeweiligen Akteuren und Akteurinnen verschwimmen lässt.

\section{Aktionsforschung!}

Methodologische Grundlage für die hier vorgeschlagene Fusion von Geographie und Aktivismus ist der Ansatz der Aktionsforschung. Dieses alternative Forschungsparadigma entstand aus dem Zusammenspiel von praktischer und theoretischer Kritik an traditionellen Formen akademischer Wissensproduktion; aus europäischen und lateinamerikanischen sozialen Bewegungen sowie kritischen Forschungsansätzen marxistischer, anarchistischer, feministischer und post-strukturalistischer Prägung.

Aktionsforschung verstehen wir als Wegweiser, kollaborative Forschungsgemeinschaften zu bilden, persönliche Beziehungen und eigene Positionierung im jeweiligen Forschungskontext so zu gestalten, dass dieser durch die Forschung gemeinsam verändert werden kann (Kindon et al.
2007, S. 13). Zentrale Bedeutung hat hierbei der zyklische Lernprozess der Aktionsforschung - bestehend aus Analyse, Planung, Aktion und Reflexion - nach dem Reflexion das Ende einer Praxisphase bildet und den Beginn der nächsten einläutet (Abb. 1).

\section{Militante Untersuchungen}

Während Militanz im Deutschen oft mit Gewalt assoziiert wird, definiert Martin Luther King Jr., die Ikone der gewaltfreien Bürgerrechtsbewegung, Militanz als ,a term of persistence, and therefore balance, rather than violence" (zit. in Bookchin et al. 2013, S. 4). Entscheidend ist hier demnach das Commitment zu langfristigem, reflektierten, das Alltagsleben der Aktivisten und Aktivistinnen prägenden Einsatz für emanzipatorische Ziele.

Der Anspruch militanter Untersuchungen ist es, die Forschung zum Werkzeug sozialer Kämpfe zu machen (Bartholl 2018). Das argentinische Colectivo Situaciones (2003) konzipiert „militancia de investigación“ als fortlaufenden Prozess der kollektiven Produktion neuer Werte und Bedeutungen, die denen der herrschenden kapitalistischen Gesellschaftsordnung Alternativen entgegensetzen. Dabei sei insbesondere (marginalisiertes) Alltagswissen mit einzubeziehen. Indem sie betonen, „forschende Militante“ müssten
Abb. 1 Der Zyklus der Aktionsforschung. (Eigene Abbildung)

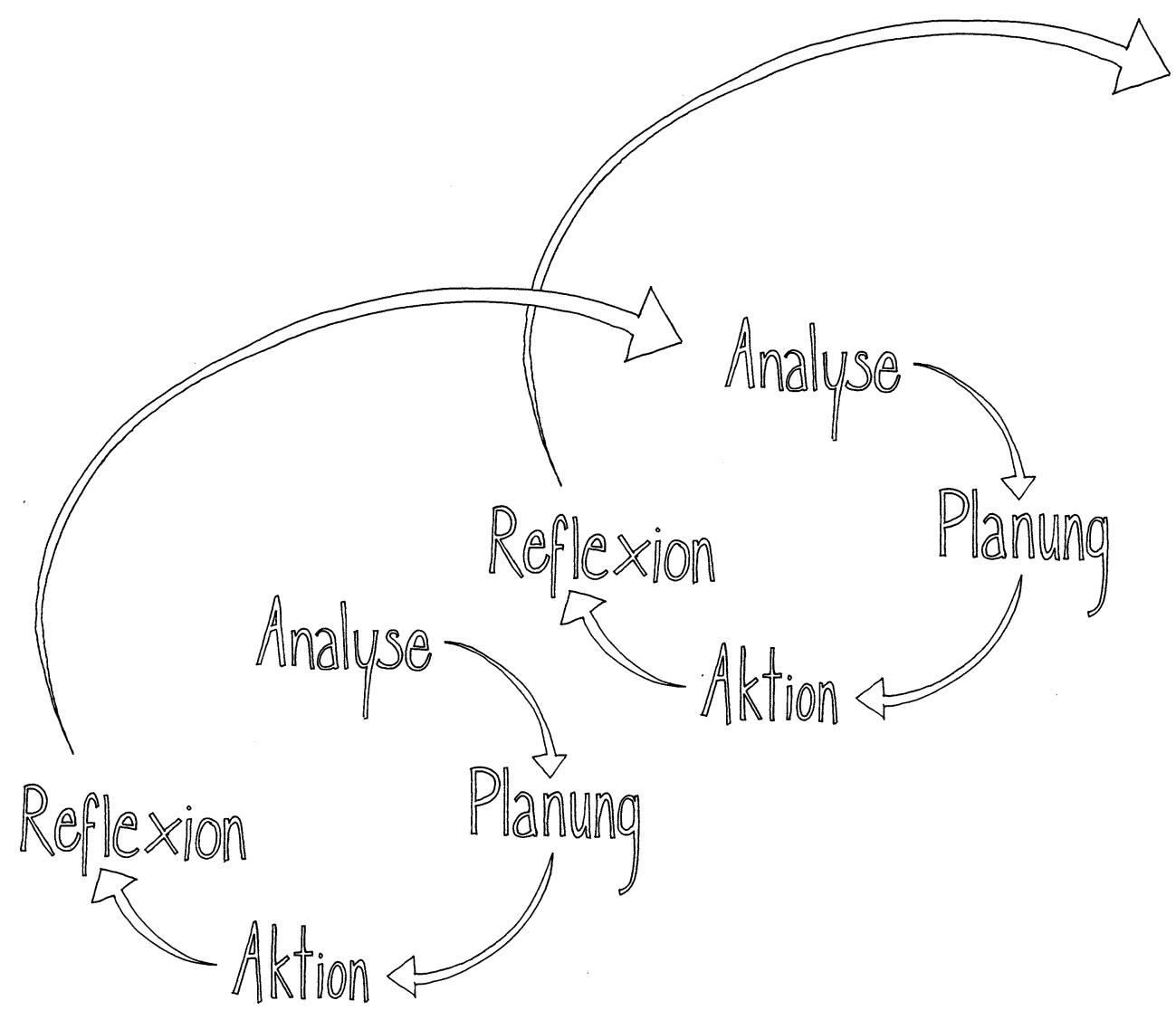


die eigene Praxis und eigene ideologische Standardantworten permanent infrage stellen, brechen sie mit etablierten Formen und Rollen des politischen Aktivismus (Counter Cartographies Collective et al. 2012, S. 446). Ziel sei eine Haltung, in der Engagement und Forschung verschmelzen:

... ways of being militants that escape the political certainities established a priori and embrace politics as research [,,research militancy“], and, at the same time, to invent forms of thinking and producing concepts that reject academic procedures, breaking away from the image of an object to be known and putting at the centre subjective experience [,militant research"] (Holdren und Touza 2005, S. 596).

Das Colectivo Situaciones versteht kollektive Forschung als Teil des politischen Kampfes. Gemeinsame Reflexion erzeuge kollektive Praxis und verleihe dieser Wirkmacht (Malo 2004, S. 35). Diese intensive Form der Wissensproduktion gehe mit einer beidseitigen Transformation der am Prozess Beteiligten einher, da die involvierten Akteure und Akteurinnen im Forschungsprozess nicht auf Distanz gingen, sondern die Nähe gesuchten. Losgelöst von der reinen Zweckorientierung könnten sie so neue, freundschaftliche und liebevolle Beziehungen aufbauen (Colectivo Situaciones 2003).

\section{Kritische und aktivistische Geographien}

Während der Anspruch, auch geographische Forschung solle Werkzeug für politische Kämpfe sein (Ribeiro de Campos 2001, S. 21) von kritischen Geographen und Geographinnen fast einheitlich erhoben wird, bleibt die Frage danach, wie konkrete Beziehungen zu sozialen Bewegungen und aktivistischen Kontexten aufgebaut und gepflegt werden, oft unbeantwortet. Während Peet (1977, S. 9) noch 1977 eine radikale Positionierung kritischer Geographen und Geographinnen im Austausch mit Aktivisten und Aktivistinnen lobte, beklagte er 20 Jahre später ernüchtert, dass dieselben Geographen und Geographinnen nur noch in exklusiven Vorträgen von der Revolution sprächen (Peet 1998, S. 68; siehe auch Piven 2010, S. 13). Kitchin und Hubbard (1999, S. 196) stellen fest: ,,... critical geographers may aim to empathize with the researched, ... but, paradoxically, they rarely join with them in their ,struggle“".

Debatten seit den 2000er-Jahren bemühen sich, das Verhältnis von Geographie und sozialen Kämpfen begrifflich neu zu fassen. „Activist geographies“, so Routledge (2009, S. 7), fordern die bestehenden Verhältnisse heraus, indem sie Theorie und Praxis in aktionsorientierten, solidarischen Kollaborationen mit widerständigen Akteuren und Akteurinnen vereinen. Pain (2003) identifiziert 3 Strömungen der aktionsorientierten Humangeographie: ,participatory research“, „combining activism and research“ und ,policy research".

Doch nicht immer hält die beanspruchte Nähe zu sozialen Bewegungen einer gründlichen Prüfung stand. Insbesondere müsse die Verteilung von Ressourcen und Privilegien zwischen Akademikern bzw. Akademikerinnen und Aktivisten bzw. Aktivistinnen problematisiert und im Sinne letzterer umverteilt werden, betonen Graziani und Shi (2020). Akademiker und Akademikerinnen bauten sich ihre wissenschaftliche Karriere ,im Fahrtwind“ von sozialen Bewegungen und aktivistischen Kontexten, so der naheliegende Vorwurf. Die Offenlegung und Reflexion der eigenen Position, unter Benennung ausbeuterischer Elemente der eigenen ,kritischen“ Wissenschaftspraxis muss als Grundlage für eine politisch-strategische Verbindung von Wissenschaft und Aktivismus auf Augenhöhe gelten. Wie Pickerill anschaulich an eigenen Erfahrungen als junge Wissenschaftlerin darstellt, geht es nicht um die Anklage „,böser“ Akademiker und Akademikerinnen zum Schutz ,armer“ Aktivisten und Aktivistinnen, sondern darum, uns von den (selbst-)ausbeuterischen Logiken neoliberaler Subjektivierung zu befreien und uns dadurch erst zur Verwirklichung des eigenen Anspruchs als aktivistische Akademiker und Akademikerinnen zu befähigen: „I would argue that we actually all need to do less, but do what we do better" (Pickerill 2008, S. 484).

\section{Bildung von unten}

Um die Praxis von Kollektiv Orangotango im Feld zwischen Aktivismus und Geographie zu verstehen, ist die Beschäftigung mit dem Verhältnis von Bildung und gesellschaftlicher Transformation entscheidend, da Bildung für uns sowohl konzeptionell als auch praktisch die Verbindung zwischen Wissenschaft und Aktivismus herstellt, oder besser, deren Grenzen verschwinden lässt. So entwickelten sich insbesondere in sozialen Bewegungen Lateinamerikas emanzipatorisch-pädagogische Praktiken, die beanspruchen, unterschiedliche Wissensformen und -vermittlungen in politische Kämpfe einzubinden. Das Pädagogische wird hier als weites Feld dialogischer Prozesse gesehen, die in soziale, spirituelle und affektive Beziehungen eingebettet sind. Kollektive Lernprozesse werden als zentral für emanzipatorische Transformation gedacht (Dinerstein und Motta 2017). Dieses Verständnis dialogischer „Bildung als Praxis der Freiheit“" (Freire 2000, S. 87-89), vermittelt durch bedeutungsvolle Alltagspraxis, ist geprägt von der von Paulo Freire konzipierten ,educação popular“ - „Bildung von unten“. In Freires Modell der „Alphabetisierung“ lernen Menschen „die Welt lesen“, um und indem sie ihr Sinn zuschreiben und sie gemeinsam gestalten. Die Rollen zwischen Lehren- 
den und Lernenden verschwimmen in Freires dialogischer Bildung.

Bildung und Forschung wiederum sieht Freire (2000, S. 109) als 2 Momente desselben Prozesses. Forschende und „Beforschte“ werden zu Mitforschenden, da Forschen kein Privileg weniger Wissender ist, sondern eine Grundeigenschaft aller Menschen. Weiterhin ist Forschung, nach Freire, kreativer Ausgangspunkt einer transformatorischen Praxis (2000, S. 181). Aktuelle Bedeutung erlangt Freire durch die Rezeption in dekolonialen (Mignolo und Walsh 2018, S. 88-90) und feministische Debatten (Hooks 2010).

\section{Für eine geographische Bildung von unten}

Inspiriert von Bell Hooks schreiben wir Kollektiv Orangotango klein - so es die Vorgaben der jeweiligen Publikation es zulassen - um die kollektive Praxis zu betonen, nicht die Personen, die Teil der vermeintlichen Gruppe sind. „kollektiv" kann so als Substantiv aber auch als Adverb funktionieren, das die gemeinsame Arbeitsweise beschreibt. Wenn im Folgenden von ,wir“ die Rede ist, meint das keine klar definierte Gruppe, sondern all jene, die in den einzelnen Prozessen mitgeforscht, mitgelernt und mitgestaltet haben, sie sind, in der gemeinsamen Praxis, Kollektiv Orangotango.

Kollektiv Orangotango entsteht von Beginn an im Überschneidungsfeld zwischen Aktivismus, Subkultur und Wissenschaft. Die vom Colectivo Situaciones geforderte Auflösung der Trennung dieser Sphären und der mit ihnen verbundenen Rollen liegt damit auch am Ausgangspunkt unserer gemeinsamen Entwicklung. Die Universität war in den 2000er-Jahren einerseits Ort der inhaltlichen Beschäftigung mit Themen wie jugendkultureller Raumaneignung, urbaner Landwirtschaft oder solidarischer Ökonomie, die uns auch in unserem eigenen Alltag beschäftigten. Ande- rerseits bot sie, im Rahmen von Austauschprogrammen, Raum für das Aufeinandertreffen mit politischer Basisarbeit und militanter Geographie in Lateinamerika, in denen wir viele jener aktivistischen Praktiken erlernten, die unsere Arbeit bis heute prägen. Eigene aktivistische und subkulturelle Betätigungen eröffneten uns Zugang zu Alltagspraktiken und -wissen, das prägend in unsere Forschungspraxis einging (s. u. a. Halder 2018). Unser neben Freire auch von der Hip-Hop-Subkultur geprägtes Bildungsverständnis hielt uns stets dazu an, auch (junge) Nichtakademiker und Nichtakademikerinnen durch gemeinsame Veranstaltungen und Aktionen in die Praxis von Kollektiv Orangotango einzubinden, die über die Jahre zu festen Bestandteilen des Kollektivs wurden.

Heute besteht unsere Arbeit aus unterschiedlichen Bildungsformaten in variierenden Kontexten entlang der sich gegenseitig befruchtenden und zyklisch überlappenden Ebenen: gemeinsam den Raum lesen lernen, alternative Repräsentationen erschaffen, Räume gestalten und widerständige Praktiken unterstützen.

Einzelne von uns sind phasenweise über Projektverträge, Lehraufträge oder Stipendien formell an Universitäten angebunden. Soweit möglich, bemühen wir uns, durch die Einbeziehung von Nichtakademikern und Nichtakademikerinnen in Veranstaltungen und Forschungsprojekte universitäre Ressourcen und Privilegien an traditionell von diesen ausgenommene Menschen umzuverteilen (Moten und Harney 2004).

Unser primäres Wirkungsfeld sehen wir allerdings auBerhalb akademischer Institutionen. Um es mit Chatterton (2008, S. 426) zu sagen: Die Themen, zu denen wir arbeiten, sind uns zu wichtig, um sie kleinen akademischen Seminaren und exklusiven Journals vorzubehalten. Ein Großteil unserer Aktivitäten sind Bildungsprozesse mit jungen Menschen (kollektive Kunstprojekte und Aktionsforschungen im öffentlichen Raum, Abb. 2) oder mit aktivistischen
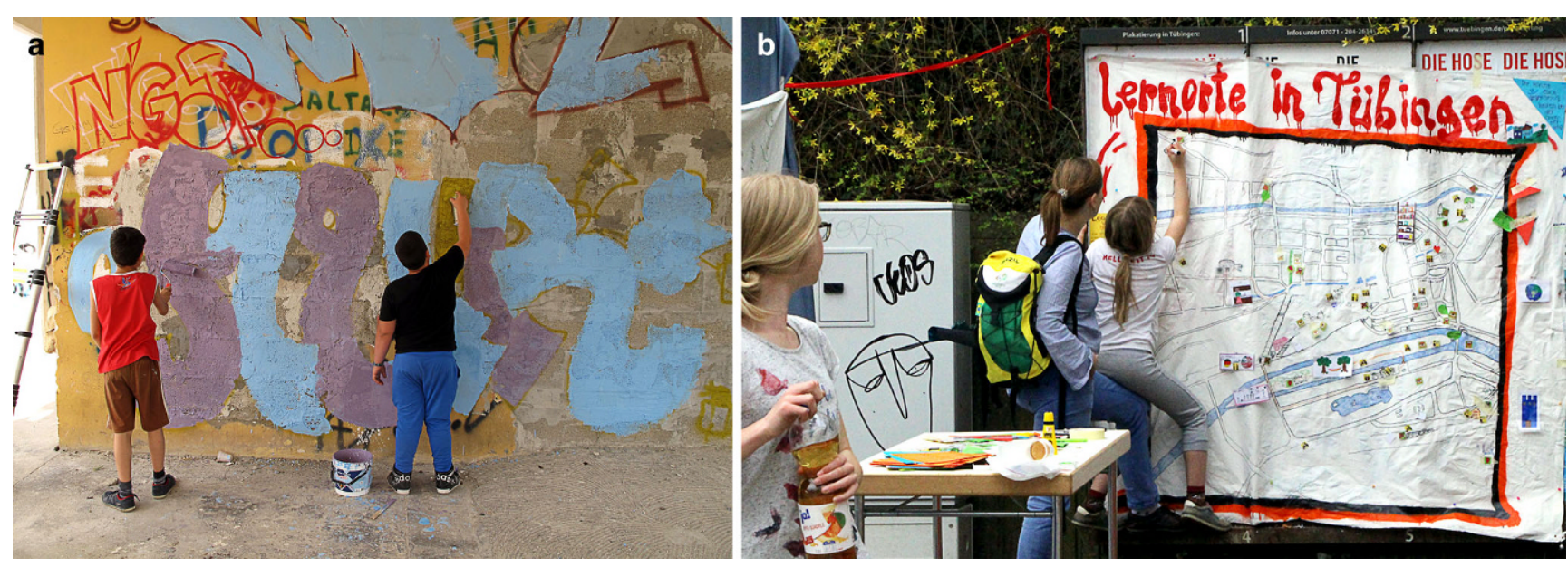

Abb. 2 Kunstprojekt und kollektive Kartierung im öffentlichen Raum. (Quelle: Kollektiv Orangotango) 

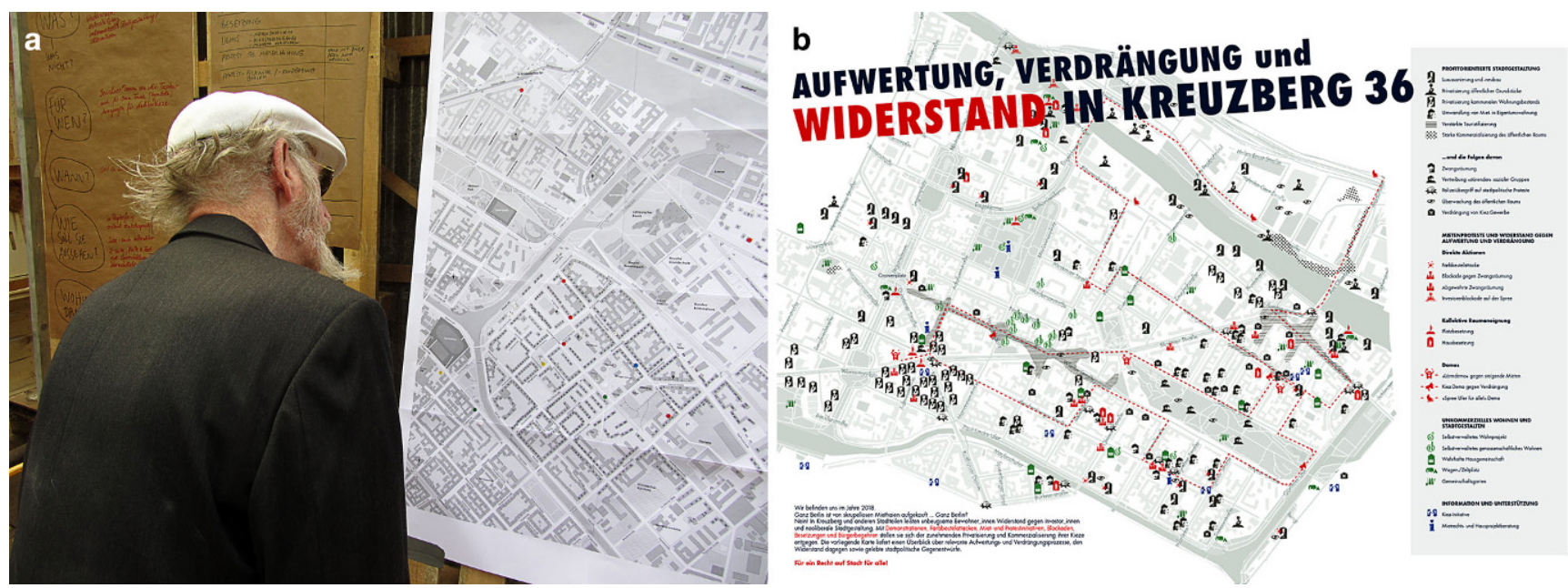

Abb. 3 Öffentliche Kartierung mit Mieter und Mieterinnen-Initiative und kritische Stadtteil-Karte. (Quelle: Kollektiv Orangotango)

Gruppen (Kartierungsprozesse sowie Reflexions- und Gestaltungsworkshops, Abb. 3).

In all diesen (Selbst-)Bildungsprozessen ,popularisieren" wir die wissenschaftliche Geographie in einer Doppelbewegung: Einerseits setzen wir Aktionsformen aus Subkulturen und sozialen Bewegungen ein, um inhaltliche Diskussionen beispielsweise zu stadtpolitischen Fragestellungen mit Akteuren und Akteurinnen zu führen, die sich sonst kaum öffentlich sichtbar zu diesen, ihr Alltagsleben unmittelbar betreffenden, Fragen äußern würden. In wochenoder monatelangen kollektiven Arbeiten an Wandbildern, die wir beispielsweise mit Geflüchteten-Initiativen oder jungen Menschen erarbeitet haben, werden deren Alltagserfahrungen mit Argumenten aus wissenschaftlichen Debatten in Bildsprache verpackt. Im Gespräch mit Anwohnern und Anwohnerinnen werden Beteiligte zu Mitforschenden, die in informellen Gesprächen und Beobachtungen Daten erheben und in die gemeinsame Reflexion einfließen lassen.

Andererseits bringen wir wissenschaftliche Methoden in außeruniversitäre Kontexte ein, um sie für kollektive Reflexion und Organisationsformen nutzbar zu machen. So nutzen wir kollektive Kartierungen als Werkzeuge der gemeinschaftlichen Sensibilisierung und Aktivierung. Kartieren also als Bildungsprozess, bei dem die eigene Beziehung zum Raum reflektiert wird, in dem verschiedene intersubjektive Perspektiven sowie verschiedene Wissensarten zusammenfließen können und sich Handlungsspielräume eröffnen. In anderen Fällen dienen wissenschaftliche Methoden der Datenerhebung und Darstellung in Online-Karten und der Vernetzung von unterschiedlichen Gemeinschaften (Abb. 4).

Wie Bell Hooks (2010, S. 19-20) in ihrer Beschreibung einer „engaged pedagogy“ ausführt, gehören Kennenlern-, Körper- und Vertrauensübungen, wie wir sie von kritischer Pädagogik und sozialen Bewegungen lernen, für uns ebenso zu diesen Prozessen, wie regelmäßige Reflexions- und Eva- luationsphasen. Nur durch die Öffnung für körperliche und affektive Praktiken und die Kultivierung gemeinsamer Werte wie Freundschaft, Demut und Fürsorge (vgl. Freire 2000, S. 89-90) können Hierarchien aufgebrochen werden. Wie vom Colectivo Situaciones (2003) gefordert, verlieren die Rollen von Forschenden und Aktivisten bzw. Aktivistinnen so ihre Bedeutung für die gemeinsame Praxis und werden durch freundschaftliche und liebevolle Beziehungen ersetzt.

Auch in unserer Publikationspraxis verbinden wir akademisches Wissen und Ressourcen mit aktivistischem Anspruch und Strategie (kollektiv orangotango+ 2018; kollektiv orangotango 2010). Zusätzlich zu thematischen Publikationen, die wir neben wissenschaftlichen Fachpublikationen auch in journalistischen Formaten publizieren, veröffentlichen wir insbesondere praxisorientierte, multimediale Materialien „zum selber machen“ (vgl. Chatterton 2008, S. 425). Im Widerspruch zu akademischen Publikationszwängen bemühen wir uns bei all unseren Publikationen um einen offenen Zugang unter restriktionsarmen Lizenzen.

\section{Commitment!}

Für unsere Praxis sehen wir im Ansatz der Aktionsforschung sowie in einer als „Bildung von unten“ verstandenen Verbindung von Wissenschaft und Aktivismus einen Weg, geographischen Aktivismus als auch aktivistische Geographie zu vereinen, dabei die Trennung zwischen Akademie und Engagement aufzuheben und neue Beziehungen zwischen Mitforschenden zu (er)leben. Prägend ist dabei die Einbettung in langjährige freundschaftlich-solidarische Netzwerke. Aus dieser Erfahrung heraus empfehlen wir einer kritischen Geographie, die den Anwendungsbezug ernst nimmt - indem sie tatsächlich den Interessen der Gemein- 


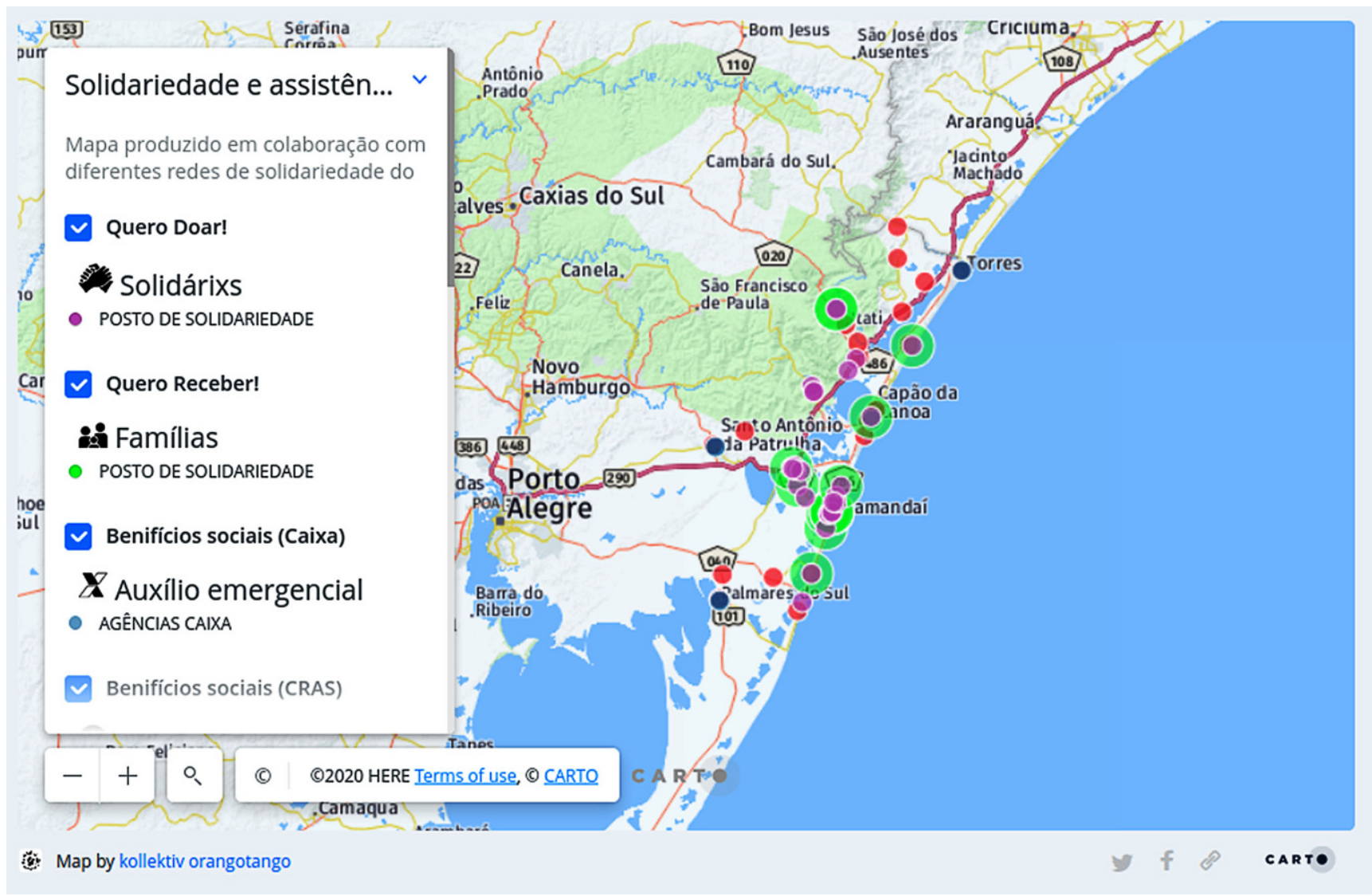

Abb. 4 Online-Kartierung zur Koordinierung von gegenseitiger Hilfe im Kontext von Covid-19, die mit regionalen Initiativen sowie Lehrenden und Studierenden der UFRGS in Südbrasilien geschaffen wurde. (Quelle: https://notanatlas.org/mapping-solidarity-in-times-of-the-covid-19/)

schaften, mit denen geforscht wird, dient -, im Sinne einer militanten Geographie ein Commitment einzugehen. Eine Verpflichtung für einen langfristigen, (selbst-)reflexiven Einsatz für soziale Bewegungen und widerständige Praktiken sowie für die Öffnung der wissenschaftlichen Geographie für andere Akteure und Akteurinnen sowie andere Wissensformen. Oder mit Milton Santos (2008, S. 261, eigene Übersetzung):

Und die Geographie, so häufig im Dienste der Unterdrückung, muss dringend neu ausgerichtet werden, um das zu sein, was sie immer sein wollte: eine Wissenschaft des Menschen.

\section{Literatur}

Bartholl T (2018) Por uma geografia em movimento-a ciência como ferramenta de luta. Consequência, Rio de Janeiro

Bookchin N, Brown P, Ebrahimian S et al (2013) Militant Research Handbook. New York University, New York

Chatterton P (2008) Demand the possible: journeys in changing our world as a public activist-scholar. Antipode 40:421-427. https:// doi.org/10.1111/j.1467-8330.2008.00609.x
Colectivo Situaciones (2003) Über den forschenden Militanten. EiPCP. https://transversal.at/transversal/0406/colectivo-situaciones/de. Zugegriffen: 30. Apr. 2020

Counter Cartographies Collective DC, Mason-Deese L (2012) Counter (mapping) actions: mapping as militant research. ACME 11:439-466

Dinerstein AC, Motta SC (2017) Introduction to the special section: social movements and social emancipation in Latin America. Bull Lat Am Res 36:3-4. https://doi.org/10.1111/blar.12525

Freire P (2000) Pedagogy of the oppressed, 30th anniversary ed. Continuum, New York

Graziani T, Shi M (2020) Data for justice: tensions and lessons from the anti-eviction mapping project's work between academia and activism. ACME 19:397-412

Halder S (2018) Gemeinsam die Hände dreckig machen - Aktionsforschungen im aktivistischen Kontext urbaner Gärten und kollektiver Kartierungen. transcript, Bielefeld

Holdren N, Touza S (2005) Introduction to colectivo situaciones. Ephemera 5:595-601

Hooks b (2010) Teaching critical thinking: practical wisdom. Routledge, New York

Kindon SL, Pain R, Kesby M (2007) Participatory action research approaches and methods: connecting people, participation and place. Routledge, London

Kitchin R, Hubbard P (1999) Research, action and "critical" geographies. Area 31:195-198. https://doi.org/10.1111/j.1475-4762. 1999.tb00083.x 
kollektiv orangotango+ (2010) Solidarische Räume \& kooperative Perspektiven: Praxis und Theorie in Lateinamerika und Europa. AGSPAK, Neu-Ulm

kollektiv orangotango+ (2018) This is not an atlas: a global collection of counter-cartographies. transcript, Bielefeld

Malo M (2004) Prólogo. In: Malo M (Hrsg) Nociones comunes: experiencias y ensayos entre investigación y militancia. Traficantes de Sueños, Madrid

Mignolo W, Walsh CE (2018) On decoloniality: concepts, analytics, praxis. Duke University Press, Durham

Moten F, Harney S (2004) The university and the undercommons: seven theses. Soc Text 22:101-115. https://doi.org/10.1215/ 01642472-22-2_79-101

Pain R (2003) Social geography: on actionorientated research. Prog Hum Geogr 27:649-657. https://doi.org/10.1191/030913250 $3 \mathrm{ph} 455 \mathrm{pr}$
Peet R (1977) Radical geography: alternative viewpoints on contemporary social issues. Maaroufa Press, Chicago

Peet R (1998) Modern geographical thought. Blackwell, Malden

Pickerill J (2008) The surprising sense of hope. Antipode 40:482-487. https://doi.org/10.1111/j.1467-8330.2008.00617.x

Piven FF (2010) Reflections on scholarship and activism. Antipode 42:806-810. https://doi.org/10.1111/j.1467-8330.2010.00776.x

Ribeiro de Campos R (2001) A geografia crítica brasileira na década de 1980 - tentativas de mudanças radicais. Geografia 26:5-36

Routledge P (2009) Activist geographies. In: Kitchin R, Thrift NJ (Hrsg) International encyclopedia of human geography. Elsevier, Amsterdam, S 7-1

Santos M (2008) Por uma Geografia Nova: da Crítica da Geografia

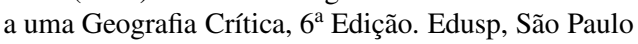

